\title{
Neutrinos from Pulsar Environments
}

\author{
A. Melatos \\ School of Physics, University of Melbourne, Parkville VIC 3010, \\ Australia
}

\begin{abstract}
Recent calculations of the neutrino fluxes and spectra from pulsar magnetospheres and wind nebulae are reviewed. The neutrinos, produced in $p p$ and $p \gamma$ collisions via pion decays, are a signature of $\mathrm{TeV}$ ions accelerated electrostatically in the magnetosphere, in the wind termination shock (Fermi), or in the wind neutral sheet (wave surfing and/or reconnection). The fluxes and spectra are related to the energy and density of the accelerated ion beam and the densities of the target species, thereby constraining ion-loaded pulsar wind models originally developed to explain the variable wisps in pulsar-driven supernova remnants. The neutrino signal may be detectable by $\mathrm{km}^{2}$ telescopes (e.g. IceCube) and is correlated with $\mathrm{TeV} \gamma$-ray emission. Related sources are also reviewed, such as early-phase post-supernova pulsar winds, pulsar-driven $\gamma$ ray-burst afterglows, and accreting neutron stars. The possibility of long baseline oscillation experiments, to search for fine splitting of neutrino mass eigenstates and non-radiative neutrino decays, is noted.
\end{abstract}

\section{Relativistic Ions}

Rotation-powered pulsars emit thermal neutrinos, via the modified URCA reaction, and non-thermal neutrinos, via the hadronic decay of relativistic protons (or heavier ions) accelerated electrically by the star. The thermal neutrino luminosity, $L_{\nu} \approx 10^{39}\left(T_{\mathrm{c}} / 10^{9} \mathrm{~K}\right)^{8} \mathrm{erg} \mathrm{s}^{-1}$, where $T_{\mathrm{c}}$ denotes the core temperature (Shapiro \& Teukolsky 1983), is emitted mainly at energies $E_{\nu} \approx k_{\mathrm{B}} T_{\mathrm{c}} \lesssim 1 \mathrm{MeV}$, below the operating band of next generation $\left(\mathrm{km}^{2}\right)$ neutrino telescopes. It is not discussed further here. The non-thermal $L_{\nu}$ depends on the ion acceleration efficiency and density of the environment. The ion source is the pulsar itself, which acts as a unipolar inductor, developing a voltage $\Phi=B \Omega^{2} R^{3} / c^{2} \approx$ $3 \times 10^{15}\left(B / 10^{12} \mathrm{G}\right)\left(\Omega / 10^{2} \mathrm{rad} \mathrm{s}^{-1}\right)^{2} \mathrm{~V}$ across its polar cap, where $B$ is the polar magnetic field and $\Omega$ is the angular frequency of the star. As $\Phi$ exceeds the work function at the surface, ions are emitted freely at the Goldreich-Julian rate $\dot{N}_{\mathrm{i}}=\dot{N}_{\mathrm{GJ}}=c \Phi / Z e$, so that the magnetospheric charge density shorts out $\mathbf{E}$ parallel to B (e.g. Arons 2003). In addition, an electron-positron $\left(e^{ \pm}\right)$outllow is generated in pair cascades in charge-starved regions near the polar cap and light cylinder (outer gap), with $\dot{N}_{ \pm}=\kappa_{ \pm} \dot{N}_{\mathrm{GJ}}$ and $10 \lesssim \kappa_{ \pm} \lesssim 10^{3}$.

Four pulsar-driven supernova remnants (plerions) have now been detected as unpulsed $\mathrm{TeV} \gamma$-ray sources by atmospheric Cerenkov imaging telescopes: the Crab (Weekes et al. 1989; Aharonian et al. 2000), PSR B1706-44 (Kifune et al. 1995), Vela (Yoshikoshi et al. 1997), and PSR B1509-58 (Sako et al. 2000). The differential energy spectrum of each object is fitted by $d N / d E \propto E^{-2.5 \pm 0.2}$ (units: $\mathrm{cm}^{-2} \mathrm{~s}^{-1}$ ), with luminosity $\lesssim 10^{-2} I \Omega \dot{\Omega}$ above $1 \mathrm{TeV}$. The $\gamma$ rays are 
unlikely to originate from the magnetosphere, as they are unpulsed. Instead, they have been attributed to hadronic processes involving accelerated ions in the pulsar wind nebula (PWN; Amato, Guetta \& Blasi 2003; Bednarek 2003), or to inverse Compton scattering if the magnetic field is below equipartition (Bednarek \& Protheroe 1997; Bednarek 2003; Guetta \& Amato 2003).

Independent evidence for ion acceleration in pulsars is provided by the arclike, sub-arsecond features (wisps) observed in the optical and X-rays in several PWNe (Hester et al. 1995, 2002; Gaensler et al. 2002). The wisps are observed to expand as circular ripples (pattern speed $\approx 0.5 c$ ), whose spacing is comparable to the Larmor radius $r_{\mathrm{L}}$ of protons with Lorentz factor $\gamma_{p} \gtrsim 10^{5}$ in the magnetic field $B_{s} \approx 0.3 \mathrm{mG}$ downstream from the termination shock in the equatorial plane of the pulsar wind. At the magnetic step of the shock, cold upstream ions gyrate forward in an $\mathbf{E} \times \mathbf{B}$ drift, compressing the frozen-in magnetic field into spikes which react-back to marshal the ions quasiperiodically into bunches. The bunches (wisps) propagate downstream for several $r_{\mathrm{L}}$ before thermalizing by absorbing self-emitted magnetosonic waves (Gallant \& Arons 1994; Spitkcovsky \& Arons 2004). The theory correctly predicts the wisp luminosity (including Doppler boosting), spacing, variability, and pattern speed for $\dot{N}_{p}=\dot{N}_{\mathrm{GJ}}, \gamma_{p}=$ $4 \times 10^{5}, \kappa_{ \pm}=10^{3}$, and $\dot{N}_{p} \gamma_{p} m_{p} c^{2} \approx 0.2 I \Omega \dot{\Omega}$ for the Crab. An alternative (steady-state) model explains the wisps as intersecting internal shocks in the backflow from a mono-polar wind (Komissarov \& Lyubarsky 2003).

\section{Neutrino and Gamma-Ray Fluxes and Spectra}

Relativistic protons yield neutrinos in two ways (Alvarez-Muñiz \& Halzen 2002): (i) inelastic collisions with an ambient cold proton, e.g. in the supernova remnant $\left(p p \rightarrow \pi^{0} \pi^{ \pm}\right.$, cross-section $\sigma_{p p} \approx 0.1 \sigma_{\mathrm{T}}$ ), where the pions share the initial energy equally; and (ii) photo-meson production via a delta resonance $\left(p \gamma \rightarrow \Delta \rightarrow \pi^{0} p\right.$ or $\pi^{0} n$, cross-section $\sigma_{p \gamma} \approx 0.01 \sigma_{\mathrm{T}}$ ), where $E_{p} E_{\gamma} \approx m_{\Delta}^{2} \approx 0.3 \mathrm{GeV}^{2}$. The neutral pions decay into $\mathrm{TeV} \gamma$ rays $\left(\pi^{0} \rightarrow \gamma \gamma\right)$, which are observable, whereas the charged pions decay into muons then neutrinos (e.g. $\pi^{+} \rightarrow \mu^{+} \nu_{\mu} \rightarrow e^{+} \nu_{e} \bar{\nu}_{\mu} \nu_{\mu}$ ). This suggests a clean observational test: if neutrinos from pulsars are produced by hadronic processes, the observed $\gamma$-ray and $\mu$-neutrino fluxes ought to be related by $\int d E_{\gamma} E_{\gamma}\left(d N_{\gamma} / d E_{\gamma}\right)=k \int d E_{\nu} E_{\nu}\left(d N_{\nu} / d E_{\nu}\right)$, with $k=1$ for $p p$ interactions and $k=4$ for $p \gamma$ interactions in the $\Delta$-resonance approximation (Alvarez-Muñiz \& Halzen 2002). The lower and upper bounds of $d N_{\gamma} / d E_{\gamma}$ and $d N_{\nu} / d E_{\nu}$ are set in terms of $E_{p}$ by the collision kinematics.

The neutrino luminosity for $p p$ collisions is given by $L_{\nu}=\frac{1}{3} \dot{N}_{p} E_{p} \min \left(1, \tau_{p p}\right)$, where the optical depth satisfies $\tau_{p p} \gtrsim 10^{-5}$ for $3 M_{\odot}$ of ejecta in the filaments of the supernova remnant (Amato et al. 2003) and one has $\dot{N}_{p} E_{p} \approx 0.2 I \Omega \dot{\Omega}$. The luminosity is reduced for $p \gamma$ collisions $\left(E_{\nu}<0.05 E_{p}\right)$, when the synchrotron or inverse Compton lifetime of $\mu^{+}$or $\pi^{+}$is shorter than the decay lifetime (Amato et al. 2003), and by flavor oscillations. The predicted neutrino event rate at Earth is given by the neutrino flux multiplied by $E_{\nu}^{-1}$ times the probability $P_{\nu \mu}$ that a neutrino converts to a muon in the detector, integrated over $E_{\nu}$ and detector area (Alvarez-Muñiz \& Halzen 2002). In the regime $1 \leq E_{\nu} / \mathrm{TeV} \leq 10^{2}$, where planned $\mathrm{km}^{2}$ detectors will be most sensitive, one finds $P_{\nu \mu} \propto E_{\nu}$. 


\section{Pulsar Magnetospheres}

Neutrino and $\gamma$-ray emission from charged heavy nuclei $N$ accelerated by magnetospheric potentials in an outer gap was analyzed by Bednarek \& Protheroe (1997). The heavy nuclei photodisintegrate via $N \gamma \rightarrow N n$ in the local MeV photon bath $\left(n_{\gamma} \approx 10^{15} \mathrm{~cm}^{-3}\right.$; Chiang \& Romani 1994), yielding $e^{ \pm}$which quench the gap potential; Fe nuclei disintegrate for $\gamma_{i} \gtrsim 10^{3}$ and He nuclei for $\gamma_{i} \gtrsim 10^{6.5}$ (Fig. 1 of Bednarek \& Protheroe 1997). The neutrons then decay to relativistic protons which collide with photons or cold protons in the supernova remnant (§2). Protons are either trapped inside the nebula and cool adiabatically, or escape as neutrons (just after the supernova) and re-enter later as protons by diffusing through the tangled magnetic field $(\sim 5 \mu \mathrm{G})$ of the remnant. From Monte-Carlo simulations with initial spin periods $5-10 \mathrm{~ms}$, nebula radii $1-2 \mathrm{pc}$, $\dot{N}_{p}=\dot{N}_{\mathrm{GJ}}$, and $\dot{N}_{p} E_{p}=0.1 I \Omega \dot{\Omega}$, Bednarek \& Protheroe (1997) predicted a $\nu_{\mu}$ flux up to $\sim 10$ times above the background for a $1^{\circ} \times 1^{\circ}$ field of view.

\section{Pulsar-Driven Supernova Remnants}

Neutrino emission from hadronic processes in Crab-like PWNe was first proposed by Berezinskii \& Prilutskii (1977) and has recently enjoyed a renaissance (Amato et al. 2003; Bednarek 2003). Guetta \& Amato (2003) estimate the proton deceleration time as $t_{p p} \approx 10^{7}\left(\rho_{\mathrm{N}} / M_{\odot} \mathrm{pc}^{-3}\right)^{-1} \mathrm{yr}$ for $p p$ collisions and $t_{p \gamma} \approx 10^{16} \mathrm{yr}$ for $p \gamma$ collisions; clearly, $p p$ collisions dominate in PWNe. However, the density and location of the target protons (and hence $t_{p p}$ ) is uncertain; they reside in a cage of Rayleigh-Taylor filaments around the PWN, whose filling factor and magnetic field structure are poorly known (Hester et al. 1996).

As a PWN ages, the $p$ and $e^{ \pm}$spectra evolve due to adiabatic and synchrotron cooling, $\nabla \mathbf{B}$ escape, and inverse Compton scattering (Amato et al. 2003; Bednarek 2003). Moreover, $I \Omega \dot{\Omega}$ decreases as the pulsar spins down, and the magnetic field evolves in concert. It is found that synchrotron losses always dominate inverse Compton, if the thermal radiation field is weak (Beall $\&$ Bednarek 2002), reducing the $p \gamma$ (but not $p p$ ) efficiency for $t_{\mathrm{N}} \gtrsim 10^{-2} \gamma_{p} \mathrm{yr}$ to less than $\approx 0.3$ (Fig. 4 of Amato et al. 2003), where $t_{\mathrm{N}}$ is the nebula age. Hence the theoretical Crab neutrino spectrum exceeds the detection threshold for IceCube and the $2^{\circ} \times 2^{\circ}$ sky background for $E_{\nu} \leq 10^{2} \mathrm{TeV}$ provided one has $\gamma_{p} / t_{\mathrm{N}} \geq 10^{4} \mathrm{yr}^{-1}$ (depending on the filling factor of the filaments; Amato et al. 2003), and the theoretical $\gamma$-ray flux for $E_{\gamma} \leq 10^{2} \mathrm{TeV}$ (where inverse Compton is appreciable; Aharonian et al. 2000) slightly exceeds existing measurements in the regime $10^{5} \leq \gamma_{p} \leq 10^{6}$ implied by wisp models (Arons \& Spitkcovsky 2004). That is, the Crab should yield $\sim 10$ events per yr in a $\mathrm{km}^{2}$ detector for $10^{4} \leq \gamma_{p} \leq 10^{7}$ (Amato et al. 2003), as should Vela and PSR B1509-58 (Guetta \& Amato 2003). However, Bednarek (2003) cautions against scaling the neutrino flux in proportion to the observed $\gamma$-ray flux without correcting for inverse Compton, and claims that Vela, PSR B1509-58, PSR J0205+6449 and PSR B1951+32 are not detectable by $\mathrm{km}^{2}$ detectors; Vela's event rate is comparable to the Crab but peaks at $<10 \mathrm{TeV}$ where the background is high. 
Relativistic protons scatter off Alfvén waves in the PWN. How important is this? Scattering requires gyroresonance, i.e. $\omega \pm \Omega_{\mathrm{ci}}-k_{\|} c=0$, with $\omega=k_{\|} V_{\mathrm{A}}$, where $\Omega_{\mathrm{ci}}$ is the ion gyrofrequency and $V_{\mathrm{A}}$ is the Alfven speed. In the Crab $\left(B=0.3 \mathrm{mG}, V_{\mathrm{A}}=10^{7} \mathrm{~cm} \mathrm{~s}^{-1}, \gamma_{p}=10^{6}\right)$, one obtains $2 \pi / k_{\|}=0.02 \mathrm{pc} \ll R_{\mathrm{N}}$ and $\omega<\Omega_{\mathrm{Ci}}$ (Alfvénic regime). The pitch-angle-averaged scattering time is then $t_{p \mathrm{~A}}=c P^{2} / 2 \pi^{2}(Z e)^{2} W_{\mathrm{a}}\left(k_{\mathrm{r}}\right)$, where $P$ is the proton's momentum and $W_{\mathrm{a}}\left(k_{\mathrm{r}}\right)$ is the energy per unit wavenumber per unit volume at the resonant wavenumber $k_{\mathrm{r}}$; that is, $t_{p \mathrm{~A}} \approx 2 \times 10^{1} \mathrm{~s} \times$ (fraction of $I \Omega \dot{\Omega}$ converted to Alfven waves).

\section{Wave-Like Pulsar Winds}

The wind of a rotation-powered pulsar is wave-like, not steady-state: the conduction current $\left(n e c \times r^{-2}\right)$ cannot shield the displacement current $\left(\Omega E / 4 \pi \propto r^{-1}\right.$ ) induced by the rotating magnetic dipole (Coroniti 1990; Melatos \& Melrose 1996). The wave can be an entropy mode, where alternating stripes of magnetic field separated by neutral sheets are convected with the flow (Coroniti 1990; Lyubarksy \& Kirk 2001), or a super/subluminal mode with a corrugated, equatorial current sheet (cf. transverse, electromagnetic oscillations in an unmagnetized plasma; Melatos 1998; Arons 2003). Like the vacuum rotator, the wind is circularly polarized along the rotation axis, where the optical/X-ray jets and knots lie in PWNe, and linearly polarized in the equatorial plane, where the wisps and torus lie (Hester et al. 2002; Gaensler et al. 2002).

Protons can be accelerated in three ways in the wind. First, they feel the ponderomotive force from the oscillating $\mathbf{E}$ in the relativistic plasma wave, attaining $\gamma_{p} \approx\left(1+e^{2} E^{2} / m_{p}^{2} c^{2} \Omega^{2}\right)^{1 / 2} \gtrsim 10^{5}$ in one wave period and implying a kinetic-dominated flow $\left(\sigma_{\mathrm{s}} \approx 10^{-3}\right)$ at the termination shock (radius $r_{\mathrm{s}}$ ), in accord with observations (Melatos \& Melrose 1996; Melatos 1998; Arons 2003). Second, in the entropy mode, protons are heated by tearing-mode reconnection in the neutral sheets (internal gas pressure $\propto r^{-8 / 3}$, external magnetic pressure $\propto r^{-2}$; Coroniti 1990) at radii $r z 2 \kappa_{ \pm} c / \Omega \ll r_{\mathrm{s}}$. Heating reaccelerates the flow and dilates the reconnection rate, so that it is incomplete at $r_{\mathrm{s}}$ (Lyubarksy $\&$ Kirk 2001). A detailed analysis of the tearing mode dynamics is needed to determine the relative efficiency of $p$ and $e^{ \pm}$acceleration. Third, if the wind of the oblique rotator approaches the split-monopole geometry of the force-free, aligned rotator $(\rho \mathbf{E}+\mathbf{J} \times \mathbf{B}=0)$, it can be shown that the wind accelerates due to $\mathbf{E} \times \mathbf{B}$ drift, with $v_{E} / c=\mathbf{E} \times \mathbf{B} / B^{2}, \gamma_{E}=\left(1+x^{2}\right)^{1 / 2}$, and $x=(r \Omega / c) \sin \theta$, where $\theta$ denotes the colatitude (Contopoulos \& Kazanas 2002). Ions surf the field lines because their (small) inertia induces a polarization drift velocity $\perp \mathbf{B}$, given by $m c \mathrm{~B} \times d\left(\gamma \mathbf{v}_{E}\right) / d t / e B^{2}$. Beyond $r \sim 10^{6} c / \Omega \ll r_{\mathrm{s}}$, where $\sigma \leq 1$, the force-free condition breaks down and ponderomotive acceleration in the wave takes over, yielding a maximum proton energy $e \Phi /\left(1+2 \kappa_{ \pm} m_{e} / m_{p}\right)$ (Arons 2003).

An appealing application of wind acceleration is to magnetars, ultramagnetized objects $\left(B \sim 10^{14} \mathrm{G}\right)$ born spinning near centrifugal break-up $(\Omega \sim$ $10^{3} \mathrm{rad} \mathrm{s}^{-1}$ ), with $\Phi \sim 10^{22} \mathrm{~V}$. Arons (2003) predicts a broken proton injection spectrum $\left(E^{-1} / E^{-2}\right.$ below/above the voltage $\sim 3 \times 10^{20} \mathrm{~V}$ when electromagnetic and gravitational wave losses balance) that escapes due to Rayleigh-Taylor disruption of the supernova remnant, with a GZK cutoff (flattening) at $10^{20} \mathrm{eV}$ at Earth if gravitational wave losses do (do not) dominate. 


\section{Alternative Sources and Applications}

Several other pulsar-driven neutrino sources have been postulated. Beall \& Bednarek (2002) argued that an early-phase supernova driven by a $10^{12} \mathrm{G}$ pulsar can accelerate Fe nuclei which then photodisintegrate due to collisions with thermal photons from the hot ejecta, giving a signal detectable by a $\mathrm{km}^{2}$ telescope above $1 \mathrm{TeV}$ within $\sim 1 \mathrm{hr}$ of birth. Similarly, Guetta \& Granot (2003) proposed a pulsar-driven $\gamma$-ray burst where protons are Fermi accelerated in mildly relativistic internal shocks $\left(d N_{p} / d E_{p} \propto E_{p}^{-2}\right)$ and $p \gamma$ collisions dominate. Anchordoqui et al. (2003) argued that accreting neutron stars (e.g. A0535+26) develop potentials $\Phi_{\mathrm{a}} \sim 10^{14} \mathrm{~V}$ in the layer between the Alfvén and inner disk radii, with target protons and $\mathrm{keV}$ photons in the disk, although they note that an improved calculation of the electrodynamics is required.

Nonthermal pulsar neutrinos may also enable new tests of the Standard Model. Pulsars offer lengthy $(\sim \mathrm{kpc})$ baselines for oscillation experiments that are sensitive to nonstandard physics, e.g. splitting of mass eigenstates, majoron processes. Moreover, by measuring the relative fluxes of $\nu_{e}, \nu_{\mu}$, and $\nu_{\tau}$ arriving at Earth, it is possible to constrain the flavor mix at the source and test for simple neutrino decay scenarios (e.g. $\nu \rightarrow$ light gauge boson) that violate CPT symmetry (Barenboim \& Quigg 2003).

Acknowledgments. I am indebted to Dafne Guetta for a lucid and comprehensive tutorial on hadronic physics in pulsar environments.

\section{References}

Aharonian, F. A., et al. 2000, ApJ, 539, 317

Alvarez-Muñiz, J., \& Halzen, F. 2002, ApJ, 576, L33

Amato, E., Guetta, D., \& Blasi, P. 2003, A\&A, 402, 827

Anchordoqui, L. A., et al. 2003, ApJ, 589, 481

Arons, J. 2003, ApJ, 589, 871

Barenboim, G., \& Quigg, C. 2003, Phys.Rev.D, 67, 073024

Beall, J. H., \& Bednarek, W. 2002, ApJ, 569, 343

Bednarek, W. 2003, A\&A, 407, 1

Bednarek, W., \& Protheroe, R. J. 1997, Phys.Rev.Lett, 79, 2616

Berezinskii, V. S., \& Prilutskii, O. F. 1977, Soviet Ast., 3, L79

Chiang, J., \& Romani, R. W. 1994, ApJ, 436, 754

Contopoulos, I., \& Kazanas, D. 2002, ApJ, 566, 336

Coroniti, F. V. 1990, ApJ, 349, 538

Gaensler, B. M., et al. 2002, ApJ, 569, 878

Gallant, Y. A., \& Arons, J. 1994, ApJ, 435, 230

Guetta, D., \& Amato, E. 2003, Astropart. Phys., 19, 403

Guetta, D., \& Granot, J. 2003, Phys.Rev.Lett, 90, 201103

Hester, J. J., et al. 1995, ApJ, 448, 240

Hester, J. J., et al. 1996, ApJ, 456, 225

Hester, J. J., et al. 2002, ApJ, 577, L49

Kifune, T., et al. 1995, ApJ, 438, 91

Komissarov, S. S., \& Lyubarsky, Y. E. 2003, MNRAS, 344, L93

Lyubarsky, Y., \& Kirk, J. 2001, ApJ, 547, 437 
Melatos, A. 1998, Mem. Soc. Ast. It., 69, 1009

Melatos, A., \& Melrose, D. B. 1996, MNRAS, 279, 1168

Sako, T., et al. 2000, ApJ, 537, 422

Shapiro, S. L., \& Teukolsky, S. A. 1983, Black Holes, White Dwarfs, and Neutron Stars (New York: Wiley)

Spitkcovksy, A., \& Arons, J. 2004, ApJ, 603, 669

Weekes, T. C., et al. 1989, ApJ, 342, 379

Yoshikoshi, T., et al. 1997, ApJ, 487, L65 